\title{
A teoria de Raymond Duval mapeada nas edições da Jornada Nacional de Educação
}

\section{Matemática}

\author{
The theory of Raymond Dyval mapped in the editions of the national Day of mathematical \\ education \\ La teoria de Raymond Duval mapeadas em lãs ediciones del dia Nacional de la Educación \\ Matemática
}

Recebido: 04/03/2021 | Revisado: 10/03/2021 | Aceito: 12/03/2021 | Publicado: 20/03/2021

Osvaldo Antônio Ribeiro Junior

ORCID: https://orcid.org/0000-0001-9512-5786 Instituto Federal de Educação, Ciência e Tecnologia do Pará, Brasil E-mail: osvaldo.ribeiro@ifpa.edu.br

Bárbara Medeiros Vieira

ORCID: https://orcid.org/0000-0002-3254-477X

Instituto Federal de Educação, Ciência e Tecnologia do Amazonas, Brasil E-mail: barbara.vieira@ifam.edu.br

Renata Gaspar da Costa

ORCID: https://orcid.org/0000-0003-1095-2075

Universidade Federal do Maranhão, Brasil

E-mail: gaspar.renata@discente.ufma.br

\begin{abstract}
Resumo
Os eventos científicos são de fundamental importância para a divulgação do conhecimento produzido pela ciência. Dessa forma, esta produção objetiva apresentar um panorama das pesquisas que utilizam a Teoria dos Registros de Representação Semiótica de Raymond Duval como aporte teórico. O evento selecionado foi a Jornada Nacional de Educação Matemática - JEM realizada pela Universidade de Passo Fundo localizada no estado do Rio Grande do Sul. Trata-se de um evento com periodicidade bienal, sua primeira edição ocorreu em 2006. Atualmente, a JEM encontra-se na sua oitava edição. As pesquisas foram analisadas de forma quali-quanti, a abordagem quantitativa foi utilizada para analisar dados como natureza da pesquisa, nível de ensino entre outros, para a categorização das pesquisas em focos e subfocos foi considerada a abordagem qualitativa, para isso foi utilizada a linha metodológica proposta por Dario Fiorentini. Foram encontrados 19 trabalhos, e foram divididos em 04 focos temáticos e 06 subfocos. Destaca-se ainda que mesmo se tratando de um evento nacional, todos os trabalhos se restringem à região sul.
\end{abstract}

Palavras-chave: Teoria dos registros de representação semiótica; Estado de conhecimento; JEM; Ensino.

\begin{abstract}
Scientific events are of fundamental importance for the dissemination of knowledge produced by science. Thus, this production aims to present an overview of research that uses Raymond Duval's Theory of Semiotic Representation Records as a theoretical contribution. The selected event was the National Day of Mathematical Education - JEM held by the University of Passo Fundo located in the state of Rio Grande do Sul. It is a biennial event, its first edition took place in 2006. Currently, JEM is in its eighth edition. The researches were analyzed in a quali-quanti way, the quantitative approach was used to analyze data such as nature of the research, level of education, among others, for the categorization of the researches in foci and subfocuses the qualitative approach was considered, for this the line methodology proposed by Dario Fiorentini. Nineteen works were found, which were divided into 04 thematic focuses and 06 subfocuses. It is also noteworthy that even though it is a national event, all works are restricted to the southern region.
\end{abstract}

Keywords: Theory of semiotic representation records; State of knowledge; JEM; Teaching.

\section{Resumen}

Los eventos científicos son de fundamental importancia para la difusión del conocimiento producido por la ciencia. Así, esta producción tiene como objetivo presentar un panorama de la investigación que utiliza como contribución teórica la Teoría de los registros de representación semiótica de Raymond Duval. El evento seleccionado fue el Día Nacional de la Educación Matemática - JEM realizado por la Universidad de Passo Fundo ubicada en el estado de Rio Grande do Sul. Es un evento bienal, su primera edición tuvo lugar en 2006. Actualmente, JEM se encuentra en su octava edición. Las investigaciones se analizaron de forma cuali-cuantitativa, se utilizó el enfoque cuantitativo para analizar datos como naturaleza de la investigación, nivel de educación, entre otros, para la categorización de las investigaciones en focos y 
subenfoques se consideró el enfoque cualitativo, por esta es la metodología de líneas propuesta por Dario Fiorentini. Se encontraron 19 trabajos, los cuales se dividieron en 04 focos temáticos y 06 subenfoques. También es de destacar que a pesar de que es un evento nacional, todas las obras están restringidas a la región sur.

Palabras clave: Teoría de los registros de representación semiótica; Estado del conocimiento; JEM; Ensenãnza.

\section{Introdução}

No início, a comunicação entre os cientistas ocorria por meio de cartas e participação em reuniões de entidades científicas para debaterem sobre o que está sendo feito em seu campo de pesquisa. Com o surgimento da especialização em diversos campos do conhecimento, surgiram também sociedades temáticas, que se reúnem em alguns eventos, que se tornaram fundamentais para a divulgação do conhecimento produzido pela ciência (Lacerda et al., 2008).

Dessa forma, o objetivo desse artigo é apresentar um mapeamento dos trabalhos lançados nos anais da JEM - Jornada Nacional de Educação Matemática, cuja temática aborda a Teoria dos Registros de Representação Semiótica de Raymond Duval.

Essa Jornada tem periodicidade bienal, sua primeira edição ocorreu no ano de 2006, é realizada pela Universidade de Passo Fundo - UPF localizada no estado Rio Grande do Sul, essa universidade realiza desde 1981 jornadas regionais de Educação Matemática. Em 2006 comemoraram os vinte e cinco anos da primeira edição das Jornadas Regionais em Educação Matemática e nessa comemoração, a equipe do Laboratório de Matemática da UPF lançou a I Jornada Nacional de Educação Matemática que se tornou um sucesso.

Sua primeira edição tinha por temática “Educação Matemática: Novos desafios! Velhas práticas?”. "A complexidade da sala de aula na contemporaneidade" foi o tema da quarta edição, realizada em 2012. A quinta edição contou com o tema "Educação Matemática: O que ensinar? Por que aprender?”. A sexta edição abordou a temática “Quais os rumos da Educação Matemática?". A sétima edição desejou agregar contribuições e discussões sobre a "Educação Matemática e Interdisciplinaridade: diálogos, experiências e práticas possíveis”. A oitava edição, realizada em 2020, teve por tema “Educação Matemática: identidade em tempos de mudança”. Esta última edição foi realizada em formato online devido à pandemia de COVID - 19.

Só a partir da sexta edição, em 2016, que os anais foram organizados em eixos temáticos que são: Eixo 1 - Políticas educacionais; Eixo 2 - Formação e desenvolvimento profissional de professores de matemática; Eixo 3 - Pesquisa em Educação Matemática; Eixo 4 - Práticas e intervenções na Educação Básica e Superior; Eixo 5 - Tendências em Educação Matemática. Os anais das edições anteriores eram divididos pelo modo de apresentação (minicursos, comunicação científica, pôster, relato de experiência, palestras, conferências, mesa redonda entre outros).

Com base nos anais da JEM foi possível realizar um mapeamento do tipo estado do conhecimento, pois se enquadra em um lócus específico, nas pesquisas que utilizam a teoria dos registros de representação semiótica como aporte teórico.

Ressalta-se que durante o mapeamento foram considerados todos os eixos temáticos e modos de apresentação.

\section{Procedimentos Metodológicos}

Esta pesquisa se configura como Estado do Conhecimento, cujo foco é mapear pesquisas relacionadas à Teoria dos Registros de Representação Semiótica de Raymond Duval, que constam nos anais da Jornada Nacional de Educação Matemática.

Segundo Ferreira (2002), as pesquisas denominadas Estado da Arte ou Estado do conhecimento possuem caráter bibliográfico e o objetivo de ambas está em mapear produções acadêmicas de um campo de conhecimento específico, 
buscando responder em que aspectos e dimensões estas pesquisas foram produzidas, exemplos de pesquisas são dissertações, teses, artigos em periódicos e anais em eventos.

As pesquisas foram analisadas de forma quali-quanti, a abordagem quantitativa foi utilizada para analisar dados como natureza da pesquisa, nível de ensino entre outros, para a categorização das pesquisas em focos e subfocos foi considerada a abordagem qualitativa, para isso foi utilizada a linha metodológica proposta por Dario Fiorentini.

Para Pereira et al. (2018) nos métodos qualitativos a interpretação sobre o fenômeno estudado parte do pesquisador, de suas opiniões, enquanto que nos métodos quantitativos os dados coletados quantitativamente são analisados através de técnicas matemáticas, como por exemplo, o uso de porcentagens.

Nesta pesquisa, o levantamento bibliográfico foi realizado no site da Universidade de Passo Fundo, local em que ocorre o evento, que disponibiliza todos os anais do JEM.

Os trabalhos foram categorizados à luz da linha metodológica proposta por Fiorentini (2002), que categoriza as pesquisas em focos e subfocos temáticos. O autor explica há diversas formas de organizar/categorizar os trabalhos, por exemplo, os estudos podem ser categorizados "pela metodologia da pesquisa utilizada ou pelo referencial teórico. Outra alternativa seria classificá-los segundo o problema ou os objetivos de investigação; ou, ainda, segundo os paradigmas epistemológicos da pesquisa educacional" (Fiorentini, 2002, p.04).

Para a seleção das pesquisas nos eventos foram considerados o título, o resumo e as palavras-chave, selecionando aqueles que faziam referência a teoria dos registros de representação semiótica de Raymond Duval.

Para a elaboração dos focos e subfocos foi realizada uma leitura interpretativa nos trabalhos.

\section{Teoria dos Registros de Representação Semiótica}

A Teoria dos Registros de Representação Semiótica (TRRS), desenvolvida pelo filósofo e psicólogo francês Raymond Duval, se enquadra na linha da Didática Francesa. Durante os anos de 1970 a 1995, Duval trabalhou no Instituto de Pesquisa em Educação Matemática - IREM em Estrasburgo, na França, onde desenvolveu pesquisas na área de psicologia cognitiva, que muito contribui para o campo da Educação Matemática. Em 1995, Duval publicou Sémiosis et pensée humaine, primeira obra sistematizada de sua teoria que representou um marco da mesma. (Freitas \& Rezende, 2013).

Duval (2012) traz dois termos importantes para sua teoria, semiósis e noésis, onde ele define semiósis como a apreensão ou produção de uma representação semiótica e noésis como a apreensão conceitual de um objeto. Logo, "não há noésis sem semiósis, é a semiósis que determina as condições de possibilidade e de exercício da noésis” (Duval, 2009, p.17).

As representações semióticas são essenciais para ter acesso aos objetos matemáticos, permitem uma "visão do objeto", uma vez que não existe acesso perceptivo, direto ou instrumental a esses (Duval, 2018; Duval, 2009; Freitas \& Rezende, 2013). Representações figurais, gráficas, em língua natural, numéricas, tabulares entre outras, são exemplos de representações semióticas (Duval, 2009; Kiefer, et al. 2020). O autor ainda afirma que uma representação semiótica enquanto explicita certas propriedades de um objeto, está também ocultando outras (Duval, 2012).

Os tratamentos e conversões são dois tipos de transformações semióticas e consideradas atividades cognitivas fundamentais ligadas a semiósis. Os tratamentos caracterizam-se por serem transformações internas, pois ocorrem no interior de um mesmo registro, isto é, mobiliza apenas um registro de representação. As conversões são transformações externas, visto que permite a passagem de um registro a outro (Duval, 2009).

A conversão desempenha um papel importante na aprendizagem, pois permite a conceitualização do objeto, entretanto essa atividade é pouco explorada no ensino e é o maior obstáculo no processo da compreensão da matemática (Duval, 2012; Duval, 2018). 
Ter acesso a muitos registros é fundamental para que o objeto não seja confundido com nenhuma de suas representações e possa ser reconhecido em cada uma dessas representações, entretanto, para garantir a apreensão conceitual dos objetos é imprescindível a coordenação entre os registros de representação (Duval, 2012).

Henriques e Almouloud (2016) definem a coordenação como sendo "a manifestação da capacidade do indivíduo em reconhecer a representação de um mesmo objeto, em dois ou mais registros distintos” (p.470). A coordenação torna-se fácil quando ocorre o fenômeno da congruência entre as representações, Duval (2009) elencou três critérios para haver congruência entre as representações, que são: “correspondência semântica entre as unidades significantes que as constituem, mesma ordem possível de apreensão dessas unidades nas duas representações, e conversão de uma unidade significante da representação de partida em uma só unidade significante na representação de chegada" p.(18).

Com base na teoria de Duval e sua importância para a compreensão dos conhecimentos matemáticos, realizou-se um mapeamento em todas as edições do evento da JEM - Jornada Nacional em Educação Matemática.

\section{Resultados e Discussão}

Considerando os critérios e procedimentos adotados, identificamos 19 artigos científicos que enfocam a teoria dos registros de representação semiótica. Ressalta-se que durante o mapeamento nos anais, a pesquisa Os registros de representação semiótica na aquisição do conceito de função afim na resolução de problemas das autoras Deise Pedroso Maggio e Rita de Cássia Pistóia Mariani consta nos anais de 2008, entretanto não é possível ter acesso ao trabalho nem por meio do site do evento nem por qualquer outro periódico. Devido a esse obstáculo, o artigo não foi considerado nos resultados desta pesquisa.

A Figura 1 evidencia o número de pesquisas em cada edição da Jornada Nacional de Educação Matemática.

Figura 1 - Quantitativo de pesquisas publicadas em cada edição da Jornada Nacional de Educação Matemática.

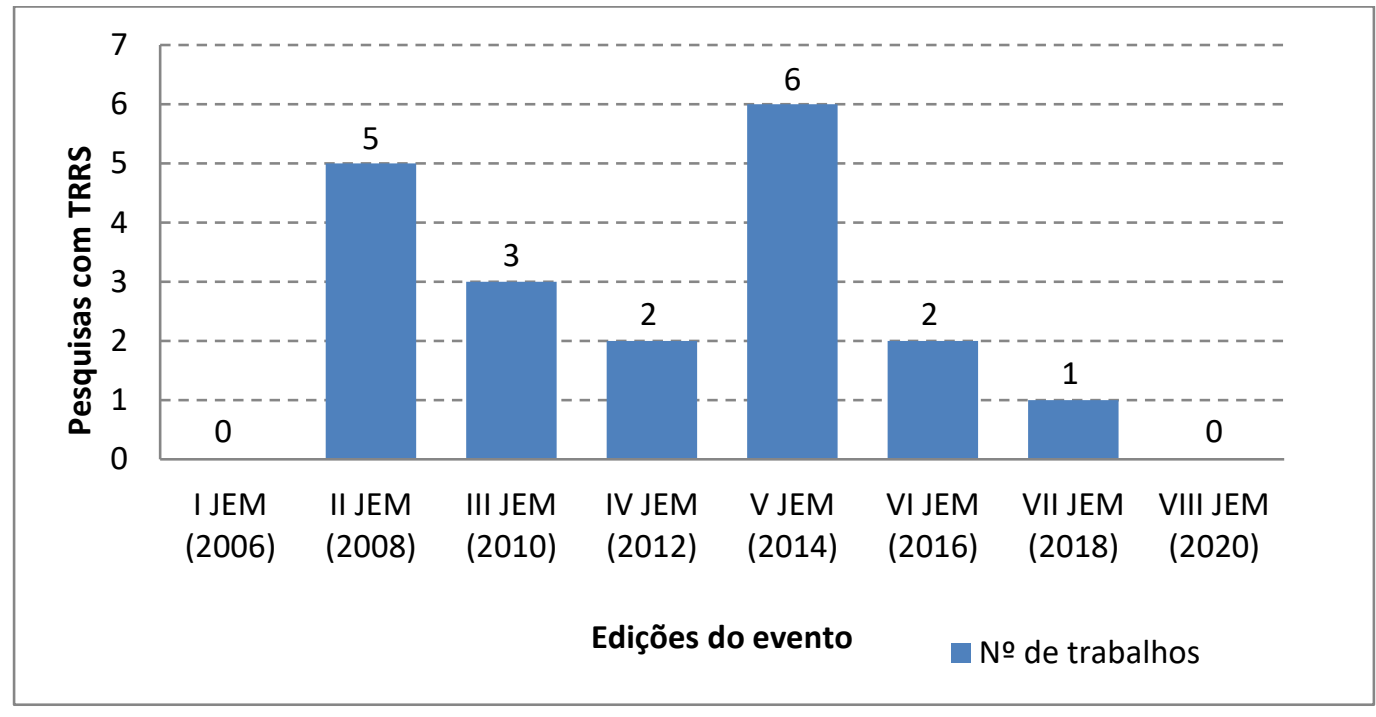

Fonte: Autores baseado nos anais dos eventos.

Destaca-se que a quantidade de trabalhos varia bastantes de uma edição a outras. A edição de 2006 e 2020 foram as únicas que não apresentaram pesquisas e não fica evidente as hipóteses que justificam a ausência de pesquisas nesses anos, da mesma forma não fica evidente porque a edição de 2014 foi a que mais apresentou resultados. 
Observou-se ainda que, apesar de ser um evento nacional, os trabalhos encontrados restringem apenas a região sul, sendo 15 pesquisas (78,9\%) localizadas no Rio Grande do Sul, 3 pesquisas $(15,8 \%)$ em Santa Catarina e apenas 1 (5,3\%) no estado do Paraná.

Quanto à natureza da pesquisa, foram divididas em pesquisas empíricas e teóricas, notou-se que 12 pesquisas, correspondendo a $63,12 \%$, possuem abordagem empírica enquanto que 7 trabalhos ou 36,84\% possuem abordagem teórica como é possível na Figura 2.

Figura 2 - Pesquisas que abordagem a teoria de Duval agrupadas por natureza da pesquisa.

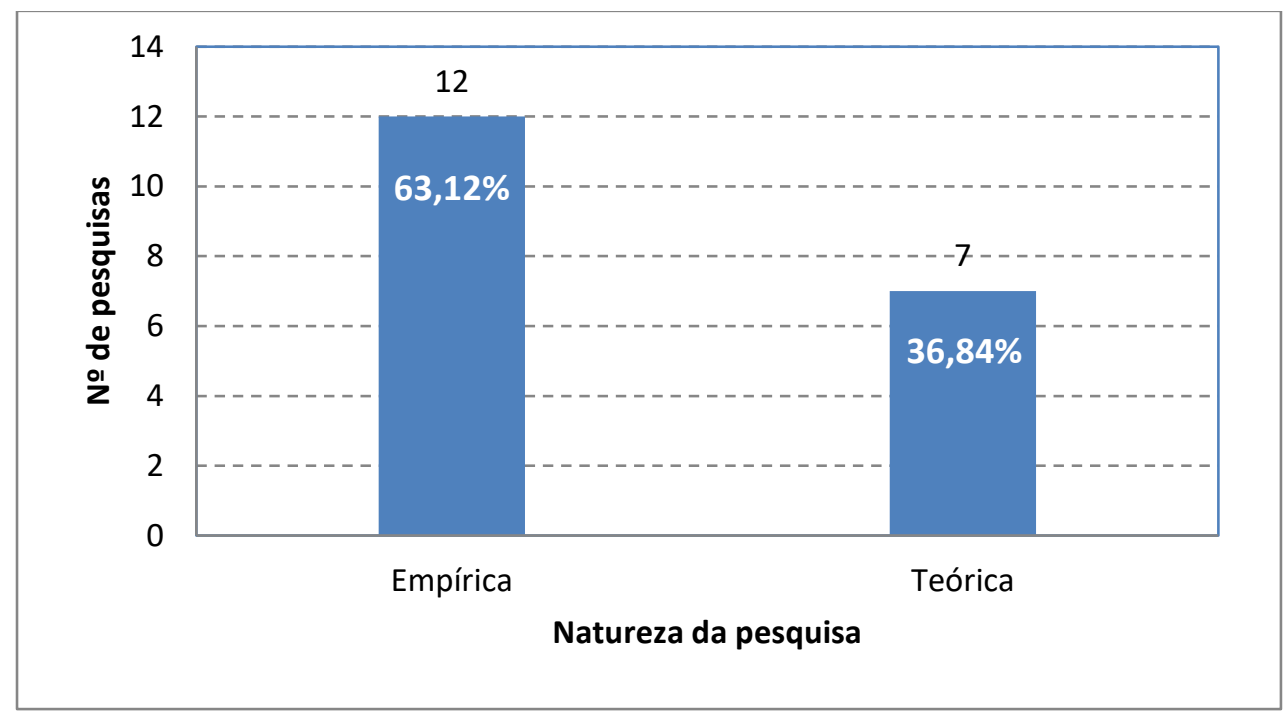

Fonte: Autores baseado nos anais dos eventos.

Na Figura 3 é possível ver a distribuição das pesquisas empíricas e teóricas ao longo das edições do JEM.

Figura 3 - Distribuição das pesquisas por natureza ao longo das edições da Jornada Nacional de Educação Matemática.

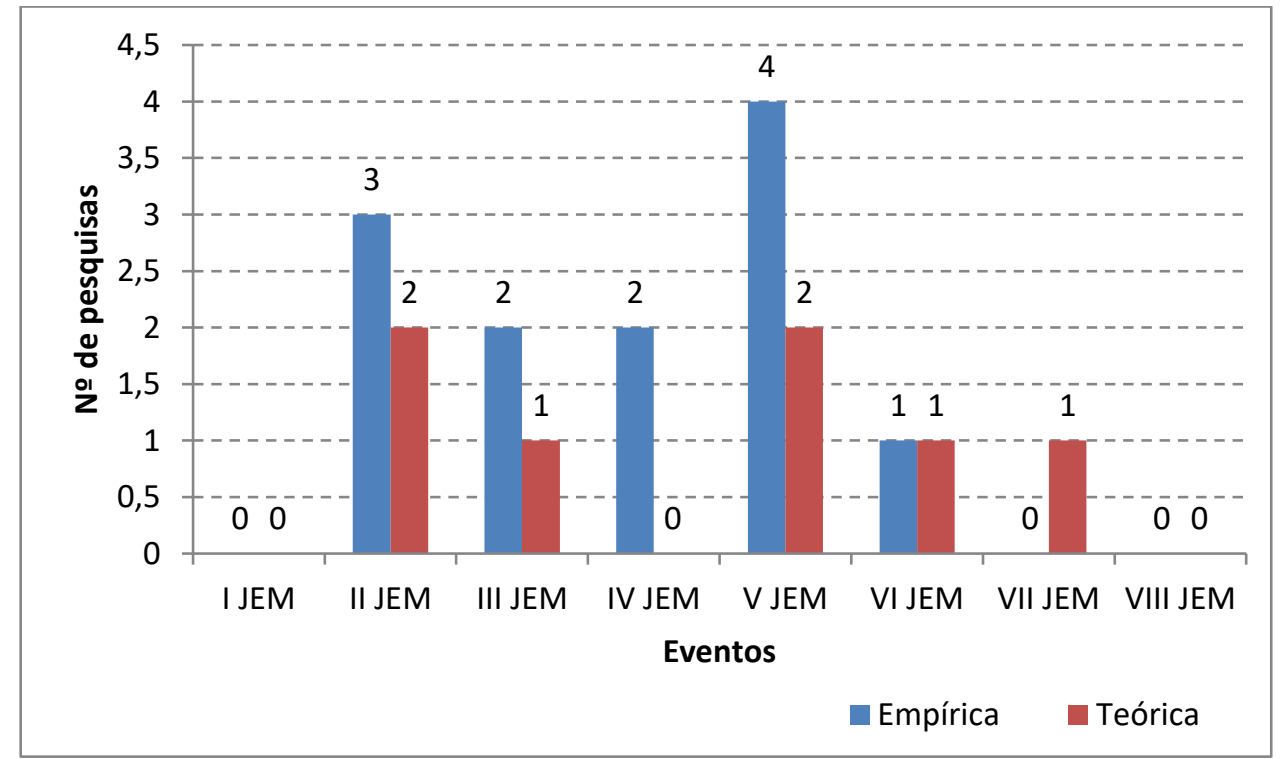

Fonte: Autores baseado nos anais dos eventos. 
Nota-se um elevado número de pesquisas empíricas na maioria das edições, apenas a VI JEM apresentou o mesmo número de pesquisas tanto para a abordagem teórica quanto para a empírica e apenas a VII JEM não apresentou abordagem empírica. Uma provável hipótese para o elevado número de pesquisas empíricas é que esta serve para validar a teoria dos registros de representação teórica, uma vez que essa teoria foi desenvolvida em um contexto educacional diferentes do brasileiro.

Outro dado analisado corresponde ao nível de ensino em que as pesquisas foram realizadas ou foram direcionadas. A Tabela 1 a seguir apresenta o quantitativo de trabalhos por nível de ensino em todas as edições do JEM que se encontrou trabalhos que abordam a TRRS.

Tabela 1 - Distribuição dos trabalhos catalogados por nível de ensino.

\begin{tabular}{c|c|c|c|c}
\hline \multirow{2}{*}{ JEM } & \multicolumn{4}{|c}{ Nível de Ensino } \\
\cline { 2 - 5 } & $\begin{array}{c}\text { Ensino } \\
\text { Fundamental }\end{array}$ & $\begin{array}{c}\text { Ensino } \\
\text { Médio }\end{array}$ & $\begin{array}{c}\text { Ensino } \\
\text { Superior }\end{array}$ & $\begin{array}{c}\text { Formação de } \\
\text { Professores }\end{array}$ \\
\hline 2008 & - & 2 & 3 & - \\
\hline 2010 & 1 & 1 & - & 1 \\
\hline 2012 & 2 & - & - & - \\
\hline 2014 & 1 & 1 & 1 & 3 \\
\hline 2016 & 1 & - & 1 & - \\
\hline 2018 & - & 1 & - & - \\
\hline Total & $5(26,3 \%)$ & $5(26,3 \%)$ & $5(26,3 \%)$ & $4(21,1 \%)$ \\
\hline
\end{tabular}

Fonte: Autores baseado nos anais dos eventos.

Os níveis de ensino forma divididos em Ensino Fundamental, Ensino Médio, Ensino Superior e Formação de professores. De acordo com os dados, percebe-se que as pesquisas na Educação Básica e no Ensino Superior estão equilibradas, e a Formação de Professores não fica muito atrás, apresentando 4 pesquisas o que corresponde a 21,1\% dos trabalhos. Andrade e Santos (2019) levantam a hipótese de que os pesquisadores ainda se preocupam mais em entender a relação da teoria com os objetos matemáticos e à medida que as pesquisas nessa vertente tornam-se saturadas, só então a Formação de professores será mais privilegiada.

Os 19 trabalhos foram categorizados por focos e subfocos temáticos conforme a linha metodológica proposta por Fiorentini (2002). Depois de categorizados, foram identificados 04 focos temáticos e 06 subfocos temáticos.

O Quadro 1 traz todos os focos e subfocos encontrados, autores pertencentes a cada subfoco e a quantidade de trabalhos em cada foco e subfoco. 
Quadro 1 - Distribuição dos trabalhos da JEM em focos e subfocos temáticos envolvendo a Teoria dos Registros de Representação Semiótica.

\begin{tabular}{|c|c|c|c|c|c|c|c|c|c|c|}
\hline \multirow[b]{2}{*}{ Foco Temático } & \multirow{2}{*}{$\mathrm{N}^{\mathrm{o}}$} & \multirow{2}{*}{ Subfoco Temático } & \multirow{2}{*}{$\mathrm{N}^{\mathrm{o}}$} & \multirow{2}{*}{ Autores } & \multicolumn{6}{|c|}{ Edições JEM } \\
\hline & & & & & 2008 & 2010 & 2012 & 2014 & 2016 & 2018 \\
\hline \multirow{4}{*}{$\begin{array}{c}\text { TRRS no } \\
\text { Ensino } \\
\text { Fundamental }\end{array}$} & \multirow{4}{*}{5} & $\begin{array}{l}\text { A TRRS como proposta } \\
\text { metodológica para o } \\
\text { processo de ensino e } \\
\text { aprendizagem }\end{array}$ & 1 & Niemann (2012) & - & - & 1 & - & - & - \\
\hline & & $\begin{array}{l}\text { A TRRS na compreensão } \\
\text { e desempenho dos alunos }\end{array}$ & 2 & $\begin{array}{l}\text { Breunig, Nehring e } \\
\text { Pozzobon (2010); } \\
\text { Niemann (2012) }\end{array}$ & - & 1 & 1 & - & - & - \\
\hline & & $\begin{array}{l}\text { A importância da TRRS } \\
\text { na aprendizagem do } \\
\text { conhecimento } \\
\text { matemático }\end{array}$ & 1 & Silva e Fuchs (2014) & - & - & - & 1 & - & - \\
\hline & & $\begin{array}{l}\text { A TRRS e a linguagem } \\
\text { matemática em Braille }\end{array}$ & 1 & Anjos e Moretti (2016) & - & - & - & - & 1 & - \\
\hline \multirow{3}{*}{$\begin{array}{c}\text { TRRS no } \\
\text { Ensino Médio }\end{array}$} & \multirow{3}{*}{5} & $\begin{array}{l}\text { A TRRS como proposta } \\
\text { metodológica para o } \\
\text { processo de ensino e } \\
\text { aprendizagem }\end{array}$ & 1 & Carpes e Santos (2018) & - & - & - & - & - & 1 \\
\hline & & $\begin{array}{c}\text { Tecnologias e TRRS no } \\
\text { Ensino }\end{array}$ & 3 & $\begin{array}{c}\text { Scheffer, Corrêa e } \\
\text { Bressan (2008); Borelli, } \\
\text { Soares, Brunger e } \\
\text { Sperandio (2010); } \\
\text { Halberstadte e Fioreze } \\
\text { (2014) } \\
\end{array}$ & 1 & 1 & - & 1 & - & - \\
\hline & & $\begin{array}{l}\text { Da realidade à sala de } \\
\text { aula e a TRRS }\end{array}$ & 1 & Severo (2008) & 1 & - & - & - & - & - \\
\hline \multirow{2}{*}{$\begin{array}{l}\text { TRRS no } \\
\text { Ensino } \\
\text { Superior }\end{array}$} & \multirow[t]{2}{*}{5} & $\begin{array}{l}\text { A TRRS como proposta } \\
\text { metodológica para o } \\
\text { processo de ensino e } \\
\text { aprendizagem }\end{array}$ & 3 & $\begin{array}{c}\text { Brandt (2008); Bueno } \\
\text { (2008); Soares e Mariani } \\
\text { (2008) }\end{array}$ & 3 & - & - & - & - & - \\
\hline & & $\begin{array}{l}\text { A TRRS na compreensão } \\
\text { e desempenho dos alunos }\end{array}$ & 2 & $\begin{array}{l}\text { Pasa; Richit e Richit } \\
\text { (2014); Roncaglio e } \\
\text { Nehring (2016) }\end{array}$ & - & - & - & 1 & 1 & - \\
\hline \multirow[b]{2}{*}{$\begin{array}{c}\text { Formação de } \\
\text { Formadores }\end{array}$} & \multirow[b]{2}{*}{4} & $\begin{array}{c}\text { Tecnologias e TRRS no } \\
\text { Ensino }\end{array}$ & 2 & $\begin{array}{l}\text { Mariani e Soares (2014); } \\
\text { Pedroso (2014) }\end{array}$ & - & - & - & 1 & - & - \\
\hline & & $\begin{array}{l}\text { A importância da TRRS } \\
\text { na aprendizagem do } \\
\text { conhecimento } \\
\text { matemático }\end{array}$ & 2 & $\begin{array}{l}\text { Moretti (2010); Silva, } \\
\text { Lacerda, Silva, Nehring } \\
\text { e Battisti (2014) }\end{array}$ & - & 1 & - & 1 & - & - \\
\hline
\end{tabular}

Fonte: Autores.

Tendo em vista o Quadro 1, nota-se que as pesquisas referentes ao Ensino Fundamental, Ensino Médio e Ensino Superior se igualaram em quantidades.

Referente ao primeiro foco intitulado "TRRS no Ensino Fundamental" notou-se que uma parcela dos autores utiliza a teoria de Duval como uma proposta metodológica para o processo de ensino-aprendizagem da Matemática, constituindo-se 
assim um subfoco que se repete em outros focos temáticos. Outro subfoco encontrado no primeiro foco foi sobre usar a teoria para compreender e avaliar o desempenho dos anos. Silva e Fuchs (2014) permitem o desenvolvimento de outro subfoco, onde a teoria é apresentada como fundamental para a aprendizagem do conhecimento matemático. O último subfoco, A TRRS e a linguagem matemática em Braille, surgiu por meio da leitura da pesquisa de Anjos e Moretti (2016). Este foco temático foi o que mais apresentou subfocos, totalizando 4 subfocos.

O segundo foco, TRRS no Ensino Médio, apresenta 3 subfocos, sendo o subfoco A TRRS como proposta metodológica para o processo de ensino e aprendizagem já mencionados no foco anterior. O subfoco Tecnologias e TRRS no Ensino é o que apresenta mais pesquisas, totalizando 3, nesse subfoco os autores propõe atividades em que os alunos utilizam softwares tais como Geogebra, Winplot, Grafeq e uso de calculadoras gráficas. Espera-se que à medida que o uso de tecnologias vá ganhando espaço na sala de aula, o número de pesquisas relacionando TRRS e tecnologia também tende a crescer.

Nota-se que esse subfoco também está presente no foco de Formação de Professores, as pesquisas presentes nesse subfoco de Formação de Professores está direcionado tanto para os professores em formação ainda tanto para aqueles que já exercem a prática. Ambientes informatizados no ensino de matemática, especificamente o uso de softwares promovem o poder da visualização e ainda "constituem um meio de transformações de todas as representações produzidas na tela" (Freitas \& Rezende, 2013, p.32).

O foco Formação de Professores além de ser o que apresenta menos trabalhos, com 4 o que corresponde a 21,1\% das pesquisas, é também o foco temático com menos subfocos, apresentando apenas 2. Como falado anteriormente, espera-se que a medida que as pesquisas voltadas para o ensino estejam saturadas, então os pesquisadores foquem mais na relação da teoria com a formação de professores.

\section{Considerações Finais}

Esta pesquisa, definida como Estado do Conhecimento, é caracterizada por realizar um mapeamento dentro do evento Jornada Nacional de Educação Matemática realizada periodicamente a cada dois anos pela Universidade de Passo Fundo, localizada no Rio Grande do Sul. Atualmente, evento possui oito edições e buscou-se mapear todas elas, buscando trabalhos que abordam sobre a Teoria dos Registros de Representação Semiótica.

Notou-se que as a I JEM e VIII JEM foram as únicas edições que não apresentaram trabalhos nessa temática. Além disso, notou-se que a quantidade de trabalhos entre o Ensino Fundamental, Ensino Médio e Ensino Superior estão equilibradas.

Fica evidente a preocupação dos autores em utilizar a teoria de Duval como uma proposta metodológica para o processo de ensino-aprendizagem e também de relacionar a teoria com uso de tecnologias, pois estes foram os dois subfocos que mais apresentaram pesquisas, cada um contendo cinco.

Futuramente, esperam-se mais trabalhos dessa temática direcionados para a formação de professores para que os docentes sintam mais confiança em utilizar a teoria nas suas aulas e compreendam a importância da conversão no processo de aprendizagem.

\section{Referências}

Andrade, A. A. \& Santos, C. A. B. (2019). Um cenário das pesquisas envolvendo a teoria dos registros de representação semiótica em edições do SIPEM. Revista de Ensino de Ciências e Matemática, 10(1), 228-245. http://revistapos.cruzeirodosul.edu.br/index.php/rencima/article/view/1922.

Anjos, D. Z. \& Moretti, M. T. (2016). A transformação semiótica da tinta ao Braille no código matemático unificado: a linguagem matemática para o estudante cego. In $6^{a}$ Jornada Nacional de Educação Matemática. 
Borelli, B. et al. (2010). Um estudo sobre o objeto matemático função e suas representações por meio do software Winplot: buscando melhorias para o ensino e aprendizagem da Matemática. In $3^{a}$ Jornada Nacional de Educação Matemática.

Brandt, C. F. (2008). Sistemas de logaritmos: representação-conceitualização. In $2^{a}$ Jornada Nacional de Educação Matemática.

Breunig, R. T. et al. (2010). Registros de representação e o ensino de álgebra: conversões realizadas por alunos da oitava série em uma situação de ensino. In $3^{a}$ Jornada Nacional de Educação Matemática.

Bueno, R. W. S. (2008). As múltiplas representações e a construção do conceito de função. In $2^{a}$ Jornada Nacional de Educação Matemática.

Carpes, P. P. G \&Santos, G. C. (2018). Uma proposta para a compreensão da função afim a partir da teoria dos registros de representação semiótica. In $7^{a}$ Jornada Nacional de Educação Matemática.

Duval, R. (2009). Semiósis e pensamento humano: registros semióticos e aprendizagens intelectuais (L. F. Levy \& M. R. A. Silveira, Trads.) Livraria da Física.

Duval, R. (2012). Registros de representação semiótica e funcionamento cognitivo do pensamento. Registres de représentationsémiotique et fonctionnementcognitif de lapensée (M. T. Moretti, Trad.). Revista Eletrônica de Educação Matemática, 7(2), 266-297. 10.5007/1981-1322.2012v7n2p266.

Duval, R. (2018). Como analisar a questão crucial da compreensão em Matemática? (M. T. Moretti, Trad.). Revista Eletrônica de Educação Matemática, 13(2), 1-27. 105007/1981-1322.2018v13n2p01.

Ferreira, N. S. A. (2002). As pesquisas denominadas "estado da arte". Educação e Sociedade, 23(79), 257-272. doi: 10.1590/S0101-73302002000300013.

Fiorentini, D. (2002). Mapeamento e balanço dos trabalhos do GT-19 (Educação Matemática) no período de 1998 a 2001. In 25aReunião Anual da Associação Nacional de Pós-Graduação e Pesquisa em Educação - ANPED, 1-17.

Freitas, J. L. M., \& Rezende, V. (2013). Entrevista: Raymond Duval e a teoria dos registros de representação semiótica. Revista Paranaense de Educação Matemática, 2(3), 10-34. http://www.fecilcam.br/revista/index.php/rpem/article/view/963.

Halberstadt, F. F. \&Fioreze, L. A. (2014). A aprendizagem da geometria analítica do ensino médio e suas representações semióticas no GRAFEQ: algumas considerações iniciais. In $5^{a}$ Jornada Nacional de Educação Matemática.

Henriques, A., \& Almouloud, S. A. (2016). Teoria dos registros de representação semiótica em pesquisas na Educação Matemática no Ensino Superior: uma análise de superfícies e funções de duas variáveis com intervenção do software Maple. Ciência \& Educação, 22(2), 465-487. 10.1590/1516731320160020012 .

Kiefer, J. G., et al. (2020). Questões de Matemática em provas de ingresso ao Colégio Politécnico da Universidade Federal de Santa Maria (2015-2020): uma análise a partir dos registros de representação semiótica. Research, Society and Development, 9(11), 1-18. 10.33448/rsd-v9i11.10177

Lacerda, A. L. et. al. (2008). A importância dos eventos científicos na formação acadêmica: estudantes de biblioteconomia. Revista ACB, 13(1), 130-144. https://revistaacb.emnuvens.com.br/racb/article/view/553.

Mariani, R. C. P \& Soares, M. A. S. (2014). A geometria analítica e a mobilização das representações semióticas na formação de professores de matemática. In $5^{a}$ Jornada Nacional de Educação Matemática.

Moretti. M. T. (2010). Teoria dos registros de representação semiótica e Educação Matemática. In $3^{a}$ Jornada Nacional de Educação Matemática.

Niemann, F. A. (2012). A problematização no ensino dos algoritmos nos anos iniciais do ensino fundamental: o tratamento das representações semióticas. In $4^{a}$ Jornada Nacional de Educação Matemática.

Niemann, F. A. (2012). O processo de ensino e aprendizagem da multiplicação: o uso dos registros de representação semiótica nos anos iniciais do ensino fundamental. In $4^{a}$ Jornada Nacional de Educação Matemática.

Pasa, B. C. et al. (2014). Representações do conceito de Derivada e Integral segundo a compreensão de estudantes de engenharia. In $5^{a}$ Jornada Nacional de Educação Matemática.

Pedroso, C. A. (2014). O procedimento informático de interpretação global aplicado às transformações de funções baseadas na idéias de Duval. In $5^{a}$ Jornada Nacional de Educação Matemática.

Pereira, A. S. et al. (2018). Metodologia da Pesquisa Científica. UFSM.

Roncaglio, V. \&Nehring, C. M. (2016). Análise de registros produzidos por estudantes de engenharia em relação ao conceito de vetor. In $6^{a}$ Jornada Nacional de Educação Matemática.

Scheffer, N. F. et al.(2008). A representação matemática com tecnologias no ensino médio: uma interação de narrativas matemáticas. In $2^{a}$ Jornada Nacional de Educação Matemática.

Severo, D. F. (2008). Os registros de representação semiótica e as frações. In $2^{a}$ Jornada Nacional de Educação Matemática. Passo Fundo - RS.

Silva, A. J. S. et al. (2014). A teoria dos registros de representação semiótica na formação de professores de matemática: um olhar para os eventos científicos. In $5^{a}$ Jornada Nacional de Educação Matemática.

Silva, M. C. P. \&Fuchs, M. J. (2014). Estágio de docência: uma análise dos registros dos alunos em processo de aprendizagem. In $5^{a}$ Jornada Nacional de Educação Matemática. 
Research, Society and Development, v. 10, n. 3, e40510313535, 2021

(CC BY 4.0) | ISSN 2525-3409 | DOI: http://dx.doi.org/10.33448/rsd-v10i3.13535

Soares, M. A. S. \& Mariani, R. C. P. (2008). Os registros de representação semiótica: contribuições para o ensino de Física e Matemática. In $2^{a}$ Jornada Nacional de Educação Matemática. 